\title{
Hadronic Final States
}

\author{
Duncan Brown ${ }^{1}$, Daniel Traynor ${ }^{2}$, Alexander Savin ${ }^{3}$ and Giulia Zanderighi ${ }^{4}$ \\ 1- University of Virginia - Dept. of Physics \\ 382 McCormick Rd, P.O.Box 400714, Charlottesville, VA 22904-4714, USA \\ 2- Queen Mary, University of London \\ Mile End, London, E1 4NS \\ 3- University of Wisconsin-Madison, \\ 1150 University Ave., Madison, WI 5370-1390, USA \\ 4- CERN - Theory Division, Geneve 23, CH-1211.
}

\begin{abstract}
In this summary we give a concise overview of the experimental and theoretical results, which were presented during the QCD and Hadronic Final State Working Group sessions at the DIS 2007 workshop.
\end{abstract}

\section{Introduction}

Final states provide a unique source of information to study elementary particle interactions. They make it possible to test our general understanding of QCD, explore QCD evolution in limiting regimes and also provide a solid reference for new physics searches. It is beyond question that precise input from the Standard Model (SM) is needed for many new physics searches that could be carried out at the upcoming LHC, most notably precise values of $\alpha_{s}$ and of the parton density functions (PDF) are needed. The role of HERA and the Tevatron in this respect is indisputable. In this document we summarize the current experimental and theoretical efforts in this direction presented at our Working Group [1].

\section{Theoretical progress}

\subsection{Higher orders and formal developments}

One of the highlights of our Working Group was the presentation of the full NNLO calculation of $e^{+} e^{-} \rightarrow 3$ jets by Aude Gehrmann [2]. The main motivation for this calculation is that the current error on $\alpha_{s}\left(M_{Z}\right)=0.121 \pm 0.001(\exp ) \pm 0.005(\mathrm{th})$ is dominated by theory and that this uncertainty largely comes from missing higher orders. A reduction of this error can be achieved by computing NNLO 3-jet event shapes and jet rates. This means computing 2-loop matrix elements with 3 partons in the final state, one-loop matrix elements with 4 partons and tree-level five parton final states. Additionally one needs to develop a formalism to cancel divergences before clustering partons into jets, this is done with a NNLO antenna subtraction [3]. After implementing results in an extended version of the program EERAD2, one can obtain a thrust distribution at NNLO for all possible color structures. The results presented show that large cancellations between the various color configurations occur, high precision results are therefore needed. This work is currently in progress, together with an implementation of other event shapes and 3-jet rates. This will allow a first NNLO determination of $\alpha_{s}$ from event shape data.

An essential ingredient in performing fixed order calculations within any quantum field theory is a method to regulate the ultraviolet (UV) divergences appearing in perturbative 
calculations. One of the most widely used schemes is dimensional regularization (DREG), where one extends the number of dimensions to $D=4-2 \epsilon$. It has long been known however that dimensional regularization breaks supersymmetry (SUSY), one of the promising new physics scenarios which might be discovered at the LHC, because the number of degrees of freedom for fermions and bosons is different. Precision calculations within SUSY are most conveniently done in a scheme which preserves the boson-fermion symmetry. An alternative to DREG is provided by dimensional reduction (DRED), where one splits the four-dimensional gluon field in a $D$-dimensional component and a so called $\epsilon$-scalar, a scalar whose coupling to fermions vanishes in four dimensions (evanescent coupling).

One attractive feature of SUSY is the unification of couplings close to the grand unification (GUT) scale. If one wants to test unification within SUSY one needs to relate a five-flavour $\overline{\mathrm{MS}}$ coupling at the electroweak scale, $\alpha_{s}^{(5), \overline{\mathrm{MS}}}\left(M_{Z}\right)$, where all heavy degrees have been integrated out (decoupled), to a full $\overline{\mathrm{DR}}$ coupling at the GUT scale, $\alpha_{s}^{(\text {full), } \overline{\mathrm{DR}}}\left(M_{\mathrm{GUT}}\right)$. To obtain a next-to-next-to-leading logarithmic (NNLL) result one needs three-loop QCD running of the $\overline{\mathrm{MS}}$ coupling from $M_{Z}$ up to $M_{\mathrm{SUSY}}$, the typical mass scale of SUSY particles, two-loop decoupling and three-loop running in SUSY from $M_{\mathrm{SUSY}}$ up to $M_{\mathrm{GUT}}$. Robert Harlander reported on this involved calculation [4]. The result turns out to be almost independent of the decoupling scale. Such a feature was also present in the Supersymmetry Parameter Analysis (SPA 05) prescription which was however based only on one-loop decoupling and one-loop running. ${ }^{\mathrm{a}}$ The difference between the two results is larger than the experimental uncertainty by almost a factor of four. A peculiar feature of the presented results is that the $\overline{\mathrm{MS}}-\overline{\mathrm{DR}}$ conversion and the $\overline{\mathrm{DR}}$ running both depend on the unphysical coupling of the $\epsilon$-scalars to quarks.

Higher order calculations are crucial in a variety of different contexts. However, at higher orders one has to deal with highly complex structures. Johannes Bluemlein reported on a method to simplify considerably physical quantities appearing in Quantum Field Theories (QFT) which depend on a single scale [5]. Single scale problems in massless or massive perturbative calculations in QFT can be expressed in terms of finite harmonic sums. These sums occur in the $\epsilon$ expansion of the integrals for higher order corrections to QCD spitting functions and Wilson coefficients for space-like and time-like processes and high-energy scattering processes such as Bhabba scattering and many others.

The idea is to perform a Mellin transform and exploit the symmetry of Feynman parameter integrals in Mellin space to perform simplifications. One derives algebraic and structural relations between multiple harmonic sums, and uses those relations to compactify considerably the result of higher order corrections. Applications of the method include the representation of $\mathcal{O}\left(\alpha_{s}^{2}\right)$ Drell-Yan and Higgs-Boson production cross-sections, anomalous dimensions and Wilson coefficients at $\mathcal{O}\left(\alpha_{s}^{3}\right)$, asymptotic heavy flavor Wilson coefficients up to $\mathcal{O}\left(\alpha_{s}^{3}\right)$, and soft and virtual corrections to Bhabba scattering. Whether the method can be extended to multi-scale problems is something to explore in the future.

At partonic level, a typical final state in small-x deep inelastic scattering off nuclei and hard proton-nucleus collisions can be characterized by the multiplicity of color-excited nucleons. Within the Reggeon field theory, each color-exited nucleon is associated with

aThis is not consistent, one-loop matching needs a two-loop running. 
the unitarity cut of the pomeron exchanged between projectile and nucleus. The pre-QCD Abramovsky, Gribov and Kancheli unitarity cutting rules formulated in the 1970s relate precisely these multi-pomeron exchange contributions to total, diffractive and inelastic cross sections.

Koyla Nikolaev explained how, starting with an exact $k_{t}$-factorization for hard pQCD in nuclear environment, a dramatic revision of the AGK rules within QCD is found [6]. Two kinds of unitarity cut pomerons emerge which describe the color excitation and color rotation inelastic interactions. The departure of the unitarity cutting rules from the ones suggested by AGK stems from the coupled-channel features of non-Abelian intranuclear pQCD. In the Reggeon field theory language, the results entail a large variety of multi-pomeron couplings which vary from one universality class for hard pQCD processes to another.

\subsection{Jets, event shapes and unintegrated PDFs}

Jets enter a variety of measurements at colliders. What a jet roughly is is quite intuitive: a bunch of particles created in a hard interaction moving around a common direction and depositing energy on their way. Theoretically, jets are tools to create a link between the hadrons measured in detectors and perturbative calculations. Given a set of four-vectors, they are fully specified by the choice of a jet-clustering algorithm, its parameters and a recombination scheme. A variety of algorithms exist (the most simple classifications are cone types and sequential recombination type algorithms), some better than others as far as infrared safety, sensitivity to higher order or to hadronization effects are concerned. Once jets are defined, a number of observables can be studied.

One interesting variable is the azimuthal correlation between two jets in QCD hard processes, $\Delta \phi \equiv\left|\phi_{j 1}-\phi_{j 2}\right|$. In the presence of only two hard partons, without additional radiation, the two jets are back-to-back, $\Delta \phi=\pi$, the non-zero azimuthal correlation provides therefore a measurement of the additional QCD radiation. The azimuthal correlation is sensitive to a variety of physical effects: it depends on the jet algorithm and recombination scheme; it is sensitive to soft/collinear gluon radiation and non-perturbative effects; according to the recombination procedure in the clustering it can be affected by so-called non-global logarithms [7] and it is one of the observables commonly used for Monte Carlo (MC) tunings. Non-global logarithms have been the subject of many studies recently. The current status is that they can be resummed numerically in the large $N_{c}$ limit with single$\log$ accuracy. Some time ago, it has been shown that the use of a jet-algorithm reduces the presence of undesired non-global logs [8], however recently it has been shown that additional logs can originate from using a jet-clustering [9]. For the azimuthal correlation the recombination scheme turns out be to decisive to establish the presence of non-global logs: if one uses a recombination scheme which adds four-momenta, as is done at the Tevatron, the observable is non-global, if on the contrary one uses an $E_{t}$ weighted average azimuth, as was done at HERA, then the observable is continuously global.

At last year's DIS workshop experimental results were presented by Magnus Hansson which did not agree with NLO 3-jet predictions [10]. Triggered by this lack of agreement between data and theory theoretical work started to improve on the theoretical accuracy. The disagreement between fixed order perturbative calculations and data is to be expected in the back-to-back region since in this region multiple soft-collinear effects become important. Yazid Delenda reported on the resummation of soft-collinear logarithms in the azimuthal 
correlation distribution in the region $\pi-\Delta \phi \sim 0$ [11]. Work in progress is now the matching with fixed NLO predictions, the inclusion of power corrections and the extension of this study to the Tevatron for which similar measurements exist.

Event-shapes provide a continuous measure of energy and momentum of the final state. Usually, they are computed perturbatively at NLO and they have large soft-collinear logarithms which can be resummed at next-to-leading logarithmic (NLL) accuracy. Additionally, they have large hadronization corrections which give rise to $1 / Q$ corrections, where $Q$ is the hard scale of the process. These corrections can be modeled in terms of one universal parameter $\alpha_{0}$. Universality implies that if one performs a combined fit of $\alpha_{s}$ and $\alpha_{0}$ then the values obtained should be independent of the event-shape used in the fit. This picture proved to work well both in $e^{+} e^{-}$and DIS. However, it has been tested only in three-jet event shapes, those, like the thrust, whose first non zero contribution comes from events with three hard partons (one incoming and two outgoing in DIS).

Andrea Banfi presented results for four-jet event shapes, whose first non-zero contribution is due to configurations with at least four partons in the event [12]. Specifically he considered $K_{\text {out }}$, roughly the radiation out of the plane spanned by the three hardest partons and the $D$-parameter, proportional to the determinant of the energy-momentum tensor, $\theta^{\alpha, \beta} \equiv$ $\sum_{h} p_{h}^{\alpha} p_{h}^{\beta} /\left(Q\left|\vec{p}_{h}\right|\right)$. Andrea presented fits of $\alpha_{s}$ and $\alpha_{0}$ from distributions of the $D$-parameter for various values of $y_{\text {cut }}$, the cut used to select events containing three hard jets. It turns out that fits from the $D$-parameter give values of $\alpha_{s}$ and $\alpha_{0}$ which are compatible with other three-jet event-shapes, where the fit region is limited to the region to the right of the peak of the distribution. For $K_{\text {out }}$ on the other hand the shape of the distribution did not allow to perform a consistent fit. A possible explanation is that the $K_{\text {out }}$ distribution is sensitive to power corrections from the four-jet region currently not included.

Final state predictions depend critically on which initial state partons enter the hard interaction and on the energy distribution of those partons. This is described by parton density functions. Standard PDFs depend on the factorization scale $\mu$ and on the longitudinal momentum fraction $x$, but they are integrated over the transverse $k_{\perp}$ component. Unintegrated parton densities (uPDFs) $\mathcal{A}\left(x, k_{\perp}^{2}, \mu\right)$ keep the information over the transverse component during the perturbative evolution. Cross sections are then given by the convolution of uPDFs with off-shell partonic cross-sections $\hat{\sigma}\left(x_{i}, k_{\perp}^{2}\right)$. If one considers e.g. the cross section for a single heavy flavoured parton as a function of $p_{t}$, because of momentum conservation at LO $p_{t}=0$, and non zero contributions appear first at NLO. If one instead uses uPDFs, the first non-zero contribution opens up already at LO. The advantage of uPDFs is therefore that by having the correct kinematics at LO one reduces considerably the size of the NLO corrections. At high energies the gluon density is dominant, therefore, as a first approximation, one can consider the contribution from gluons uPDFs only.

The $\mathrm{uPDF}$ is determined by a convolution of a non-perturbative starting distribution $\mathcal{A}_{0}\left(x, \mu_{0}\right)$ and the CCFM evolution $[13] \tilde{\mathcal{A}}\left(x, k_{\perp}, \bar{q}\right)$

$$
x \mathcal{A}\left(x, k_{\perp}, \bar{q}\right)=\int d x^{\prime} \mathcal{A}_{0}\left(x^{\prime}\right) \frac{x}{x^{\prime}} \tilde{\mathcal{A}}\left(x, k_{\perp}, \bar{q}\right),
$$

where the distribution $\mathcal{A}_{0}$ is parametrized at a starting scale $\mu_{0}$ by

$$
x \mathcal{A}_{0}\left(x, \mu_{0}\right)=N x^{-B_{g}}(1-x)^{4} .
$$


Hannes Jung presented results of fits of $B_{g}$ from inclusive $F_{2}$ and from semi-inclusive $F_{2}^{c}$, giving values of $B_{g}=0.028 \pm 0.003$ and $B_{g}=0.286 \pm 0.002$ respectively (for $\mu_{0}=1.2 \mathrm{GeV}$ ) [14]. The different value of $B_{g}$ from $F_{c}^{c}$ changes the uPDF significantly, since the discrepancy is not covered by the experimental uncertainty. To resolve the discrepancy one can perform a fit to DIS dijet cross sections. The result for $B_{g}$ turns out to be very similar to the one obtained from $F_{2}$. Regarding the residual discrepancy with the determination of the $x$ component of $\mathcal{A}_{0}$ from $F_{2}^{c}$ one can observe that the rise of the gluon distribution from $F_{2}^{c}$ comes from lowest $x$ points. If one considers only $x>2 \cdot 10^{-4}$ and uses the $F_{2}$ value of $B_{g}$ in the $F_{2}^{c}$ distribution one obtains a $\chi^{2} /$ d.o.f. $\sim 1.1$. Finally, Hannes presented the first measurement of the intrinsic $k_{t}$ component of $x \mathcal{A}\left(x, \mu_{0}\right)$ assuming a Gaussian distribution $\sim \exp \left(-\left(k_{t 0}-\mu\right)^{2} / \sigma^{2}\right)$. The fit to the intrinsic $k_{t}$ distribution, presented at this workshop for the first time, gives $\sigma \sim 1.5$ and $\mu \sim 1.5$ and is consistent with a Gaussian distribution, although other distributions are not excluded. One additional remark about uPDFs is that one can describe the $\Delta \Phi$ distribution between jets with Cascade using the new fit for the uPDFs.

While NLO predictions for inclusive jet spectra typically have errors $\sim 10-20 \%$, NLO predictions for heavy jets ${ }^{\mathrm{b}}$ fare much worse, the uncertainty being around $\sim 40-60 \%$. The NLO prediction for heavy-jets is thus a poorly constrained NLO calculation. Additionally, the experimental errors of the measurement at the Tevatron are smaller than the corresponding theoretical uncertainty. To understand the reason for the large theoretical uncertainty it is useful to examine the flavour production mechanisms. At LO the only production mechanism for heavy flavour is flavour creation (FC), $l l \rightarrow h h$, where $l$ is a generic light flavour and $h$ denotes the heavy flavour under study. At NLO on the other hand two other processes enter, flavour excitation (FEX), $l h \rightarrow l h$, where the incoming heavy quark stems from a collinear splitting of an incoming gluon, and gluon splitting (GSP) $l l \rightarrow l(l \rightarrow h h)$. Since the NLO processes are enhanced by collinear and soft logarithms, $\ln \left(p_{\mathrm{t}, \mathrm{jet}} / m_{Q}\right)$ (where $m_{Q}$ denotes the mass of the heavy quark), they turn out to be more important that the LO contribution. This means that a NLO calculation, which treats FC at NLO, but where FEX and GSP enter only at LO has very large K-factors $(\sim 5$ at the Tevatron and $\sim 8$ at the LHC, for accessible $p_{t}$ values) and therefore also very large scale dependencies.

Giulia Zanderighi showed that if one instead clusters events using an infrared-safe, flavour algorithm, which takes into account the difference between quark and gluon QCD production, then flavour becomes an infrared safe quantity. This implies that no logarithms $\ln \left(p_{\mathrm{t}, \text { jet }} / m_{Q}\right)$ are present and one can accordingly perform a light-quark calculation e.g. with NLOJET $++[15]$. This gains roughly a factor 3 in accuracy: $\mathrm{K}$ factors become $\sim 1.3$ and scale variation uncertainties are around $10-20 \%$, as is to be expected from a true NLO calculation [16]. The method however requires a good understanding of single and double $b$-tagging efficiencies, and further experimental investigation in this direction is needed.

\subsection{Parton showers and matrix element event generators}

QCD Monte Carlo (MC) generators and simulations are vital for physics at the LHC. It is therefore crucial to examine critically different MC components, including perturbative aspects (parton showers, matrix element corrections, matching), non-perturbative aspects

${ }^{\mathrm{b}}$ Experimentaly a heavy-jet is any jet containing at least one heavy tagged object. 
(hadronization, underlying event) and tuning of the event generators. Parton showers (PS) reflect our understanding of pQCD to all-orders. They are commonly believed to capture at least the leading logarithmic structure. Observables sensitive to radiation in a limited phase space region (energy flow distributions, event shapes, ... ) are sensitive to non-global logarithms even at leading order. Is it therefore important to re-examine the accuracy of showers in these instances in order to establish if for example leading non-global logarithms are erroneously tuned in the MC parameters.

The starting point of the study presented by Mrinal Dasgupta is the fact that angular ordering $(\mathrm{AO})$ catches the relevant part of these non-global logarithmic corrections. HERWIG, therefore, which is based on angular ordering is expected to be close to the full resummation of the non-global logarithms (known only in the large $N_{c}$ limit). PYTHIA (before v.6.4) uses as an ordering variable, the virtuality $\mathrm{m}^{2}$ and rejects a posteriori configurations which do not respect AO, and is expected to do worse. PYTHIA v.6.4 on the other hand, like ARIADNE, is based on dipole phase space, and should have the full leading logarithmic behaviour. In the study presented by Mrinal Dasgupta these expectations are tested by comparing full resummed results matched to NLO with predictions for parton showers for the radiation in a slice in rapidity $\Delta \eta$ as a function of $E_{t}$, the total transverse energy of hadrons in the rapidity slice [17]. It turns out that the above expectations are confirmed unless one takes a quite large region of pseudorapidity, e.g. $\Delta \eta=3$. For large rapidity slices a large discrepancy is found between the new PYTHIA (v.6.4) and the resummed result. Further studies are needed to clarify this discrepancy and to generally understand the various parton showers in a quantitative fashion. In general, it has been stressed that whenever possible one should compare results from HERWIG and PYTHIA, and when possible of other Monte Carlos.

As stressed above, Monte Carlos today are indispensable tools for many experimental studies. One of their very conventional application is to estimate non-perturbative (NP) effects. NP effects at hadron colliders include both final state hadronization effects and effects due to the underlying event (UE). Single jet inclusive distributions, parametrized in terms of a jet-radius $R \equiv \sqrt{(\Delta y)^{2}+(\Delta \phi)^{2}}$ have $1 / p_{t, \text { jet }}$ power corrections due to the hadronization and UE.

Lorenzo Magnea [18] explained that it is possible to show analytically that hadronization corrections are distinguishable from UE because they exhibit a singular dependence on $R$, while UE effects are proportional to the radiation they collect in a given region and so scale as $\sim \pi R^{2}$. Lorenzo then presented a MC study where one runs a MC at parton level (p), hadron level without UE $(\mathrm{h})$ and with UE $(\mathrm{u})$. One then selects events with two hard jets with the hardest jet in a chosen $p_{t}$ range and one defines for each hadron level a measure of the size of the power correction

$$
\Delta p_{T}^{(h / u)} \equiv \frac{1}{2}\left(p_{T, 1}^{(h / u)}+p_{T, 2}^{(h / u)}-p_{T, 1}^{(p)}-p_{T, 2}^{(p)}\right), \quad \Delta p_{T}^{(u-h)} \equiv \Delta p_{T}^{(u)}-\Delta p_{T}^{(h)}
$$

One can examine $\Delta p_{t}^{(h / u)}$ divided by the leading behaviour in $R(1 / R$ for hadronization effects and $R^{2}$ for UE) for two different partonic channels, e.g. a quark dominated channel, $q q \rightarrow q q$, and a gluon dominated one, $g g \rightarrow g g$, both for HERWIG and PYTHIA. With HERWIG the result of this operation is a flat distribution as a function of $R$, signaling that the leading $R$ dependence has been correctly extracted. Additionally, one can see that 
the size of the UE is independent on the hard partonic scattering channel. With PYTHIA on the contrary the UE seems to "know about the hard scattering" i.e. instead of a flat distribution one has a leftover $1 / R$ dependence. This is perhaps surprising. Additionally the $\mathrm{UE}$ is sensitive to the hard channel (increases when going from quarks to gluons). A correct modeling of the UE is the basis for precision phenomenology at the LHC, since for example this input enters into the determination of the jet energy scale. These types of discrepancies should therefore be addressed soon. The work presented here is based on disentangling hadronization from UE effects by exploiting their different $R$ dependences. It was therefore recommended that, whenever possible, measurements for different values of the jet-radius $R$ should be provided.

In a more general context parton showers rely on the universal soft and collinear (SC) factorization of the QCD matrix elements. This is a universal property and is true to all perturbative orders. In an ideal world this should be the only approximation made in MCs. However, practically all current MCs are subject to many other approximations, e.g. interference diagrams are treated only approximately with angular ordering, the color algebra is valid only in the large $N_{c}$ limit, spin treatment is usually inexact, other first approximations are carried in order to simplify phase space and further arbitrary techniques are often employed.

Zoltan Nagy reported on ongoing work to formulate a parton shower which removes all the above approximations [19]. The method is based on recursive equations that can be used to generate a lowest order parton shower for hard scattering in hadron-hadron collisions. The formalism is based on the factorization of soft and collinear interactions from harder interactions. It incorporates quantum interference between different amplitudes in cases where the interference diagrams have leading soft or collinear singularities. It incorporates the color and spin information for the hard partons. One of the motivations for this more formal development of a parton shower is to have a method that can be merged to NLO calculations in a natural way.

While parton showers are crucial for many studies, it is also well-known that in many cases parton showers fail dramatically. This is for instance the case in events with many hard jets: a parton shower has the full matrix element only for the primary hard scattering involving few partons, while all other emissions are treated in the soft-collinear approximation. ${ }^{c}$ Therefore, any study which looks at properties of events with many hard well-separated particles in the final state should be based on tools which go beyond the soft/collinear approximation of parton showers. Today tree level matrix element generators (ALPGEN, Helac, MadGraph/MadEvent, Amegic,$++ \ldots$ ) exist which can treat according to the process up to around 5-8 particles in the final state including full spin correlations/interference. Simon Visscher reported on progress in Madgraph/MadEvent (MG/ME) [20]. Specifically, MG/ME have now two different matching schemes, an MLM type based on event rejection after the PS and a CKKW type based on Sudakov reweighting and vetoing on showers. This work set a basis for a systematic comparison between different generators, matching techniques and shower algorithms. [21]

\footnotetext{
${ }^{c}$ Traditionally, using the soft/collinear approximation means that there is a deficit of radiation in the large angle region. This is however not always the case, one can enhance the shower arbitrarily so as to fill, even overfill, those regions, but this is artificial and ad-hoc.
} 
As an example Simon presented results obtained with MG/ME for $t \bar{t}+0,1,2,3 \ldots$ jet events. These processes are important for a variety of reasons. In addition to their interest per se, they are a background to $t \bar{t} H$ production and in two-Higgs Doublet Models (2HDM) $p p \rightarrow W^{+} W^{-} b \bar{b} b \bar{b}$ could be the most interesting channel to discover the charged Higgs. In this case one needs a reliable $t \bar{t}+0,1,2,3 \ldots$ jet event sample. If one looks at the rapidity distribution of the hardest jet, predictions obtained with Pythia are depleted of radiation in the central rapidity region. Full matrix element based predictions are not, showing the need to go beyond SC approximation of parton showers for this type of analysis.

Despite the fact that QCD and EW corrections are nothing but higher order corrections dictated by the same SM Lagrangian, traditionally the two corrections have mostly been treated separately. Alessandro Vicini reported on a calculation of combined QCD $\left(\mathcal{O}\left(\alpha_{s}\right)+\right.$ PS) ) and EW $\left(\mathcal{O}\left(\alpha_{\text {ew }}\right)+\right.$ PS) corrections to charged current Drell-Yan (DY) processes at hadron colliders [22]. DY processes are unique at hadron colliders. The presence of high $p_{t}$ leptons, together with the large cross section makes their detection very easy. DY processes are useful for validating pdfs, as luminosity monitors or to obtain precision measurement of EW observables $\left(M_{W}, \Gamma_{W} \ldots\right)$. $W / Z$ production in association with jets is an important background to SM and beyond Standard Model (BSM) signals, especially to new gauge bosons. In pure QCD the state-of-the-art are fully differential NNLO predictions exist [23].

Alessandro Vicini presented results obtained with the HORACE event generator, which includes state of the art EW radiative corrections to DY processes including exact $\mathcal{O}\left(\alpha_{\text {ew }}\right)$ radiative corrections matched to multiple photon radiation via PS. It's a fully exclusive event generator, which can be easily interfaced to QCD showering programs like HERWIG, events are saved in a Les Houches compliant form and it can be interfaced to the LHAPDF package. The QCD and EW corrections are matched with an approximate additive procedure, which works to $\mathcal{O}\left(\alpha_{s} \alpha_{\text {ew }}\right)$ as long as hard non-collinear radiation effects are not important. Beyond the additive approximation a full two-loop $\mathcal{O}\left(\alpha_{s} \alpha_{\text {ew }}\right)$ calculation is needed. Results presented for $M_{T}^{W}$ and $p_{\perp}^{l}$ show that around the jacobian peak positive QCD corrections compensate negative EW corrections and that EW effects are mandatory in order to extract $M_{W}$, only QCD PS are not sufficient. Another interesting QCD-EW interplay effect lies in the fact the convolution with QCD PS modifies the relative size and shape of the EW corrections. Due to the presence of EW logarithms, EW effects become important in the tails of distributions $\left(M_{T}^{W} \sim 2 \mathrm{TeV}, p_{\perp}^{\mu} \sim 1 \mathrm{TeV}\right)$ they can amount to up to $\sim \mathcal{O}(30) \%$ corrections, however cross-sections are very tiny in those regions.

\section{$3 \quad$ Particle production in $e e, e p, p \bar{p}$ and $p p$ collisions}

\subsection{Pentaquark searches}

In 2004 a comprehensive program to search for pentaquarks (PQ) in high statistic, high resolution experiments was started by the CLAS collaboration at Jefferson Lab. P. Rossi (CLAS) presented recent results for the four photoproduction channels studied so far [24]. No evidence for a PQ signal was observed. From these results CLAS set an upper limit on the $\mathrm{PQ}$ production cross section on protons and neutrons.

M. Del Degan (H1) presented the invariant mass spectrum of the $\Xi \pi$ system, studied using deep inelastic scattering (DIS) data collected with the H1 detector at HERA [25]. A clear signal for the well established unexotic $\Xi(1530)^{0}$ baryon is observed. Despite having 
similar statistics as NA49, the $\Xi^{--/ 0} \mathrm{PQ}$ signal could not be confirmed. Upper limits for $\Xi^{--/ 0} \mathrm{PQ}$ production from $\mathrm{H} 1$ are in agreement with those published by the ZEUS collaboration.

\subsection{Deuteron and antideuteron production}

A first measurement of the production of deuterons and antideuterons in DIS was presented by S. Chekanov (ZEUS) [26]. It is interesting to mention that this is the first measurement of deuteron production in elementary particles collisions. Production rates of (anti)deuterons are significantly reduced relative to the production of (anti)protons, consistent with other world measurements. The production rate of deuterons was measured to be approximately three times larger than for antideuterons. The production rates were also studied in terms of the coalescence model.

\subsection{Exclusive final states in $e^{+} e^{-}$}

BaBar has now taken over $400 \mathrm{fb}^{-1}$ of data and has used this data in a variety of tests of QCD using different production channels. The first observation of $e^{+} e^{-} \rightarrow \rho^{0} \rho^{0}$ and $e^{+} e^{-} \rightarrow \phi \rho^{0}$ was reported by S. Saremi (BaBar) [27]. The final states for these channels are even under charge conjugation. Such processes can be understood in terms the TwoVirtual-Photon Annihilation model. Other results presented were a new test of factorization in the $\bar{B} 0 \rightarrow D^{*}+\omega \pi^{-}$channel, for which there is good agreement, and a measurement of the $\eta$ and $\eta^{\prime}$ transition form factors, were the ratio of the form factors is inconsistent with theoretical predictions.

\subsection{Identified particle measurements in $e p$ and $p p$}

Recent results on strange particle production $\left(K^{ \pm}, K_{S}^{0}, \Lambda^{\prime} s\right)$ and Bose-Einstein correlation's (BEC) between kaons were presented in a talk given by B. Levchenko (ZEUS) [28]. The parameters for the BEC agree well for different kaons and are consistent with $\mathrm{H} 1$ and $e^{+} e^{-}$ results. No sizeable barion-antibarion asymmetry was observed. The ratio of baryons to mesons in the resolved photoproduction regime is much larger than in $e^{+} e^{-}$and is not described by Pythia predictions.

This observation agrees well with similar results from $p p$, presented by M. Heinz (STAR) [29]. STAR also presented the $p_{T}$ spectra for different particles, which for the first time can be described by the NLO pQCD predictions due to improved fragmentation functions (FF) for baryons and strange particles using the light-flavour separated measurements in $e^{+} e^{-}$ collisions from OPAL.

\subsection{Fragmentation functions at HERA}

The scaled momentum spectra in the current region of the Breit frame in DIS $e p$ scattering at HERA was measured by ZEUS, presented by B. Brzozowska, with high precision data covering a large range of energy scale (5 to $170 \mathrm{GeV}$ ) [30]. The NLO pQCD theoretical predictions cannot reproduce the data in the entire phase space and cannot describe the $Q^{2}$ evolution of the $x_{P}$ distribution. Differences between predictions using different FF were found to be small. 


\subsection{Particle production in jets}

The relative roles of perturbative and non-perturbative QCD in the development of jets have been studied by CDF. In his talk L. Pinera presented the momentum distributions and multiplicity of charged particles in jets, momentum correlations of particles and their $k_{T}$ distribution [31]. The energy scale range covered in this analysis goes from 20 to $160 \mathrm{GeV}$. The data are generally well described by pQCD within MLLA approach, but at large $k_{T}$ the theory predictions significantly deviates from data.

\section{Particle production in collisions with heavy-ions}

\subsection{Property of the Quark-Gluon Plasma}

Hadronic final states and their correlations were used by the PHENIX collaboration (talk presented by C. Ogilvie) to investigate properties of the Quark-Gluon Plasma (QGP) and it's impact on particles passing through it [32]. Energy loss and meson suppression deliver information on the density of QPG while direct photons are insensitive to the QPG. These properties can aid the comparison of collisions of protons with that of heavy-ions. The broadening of jets can be explained in terms of induced gluon radiation in the QGP. High $p_{T}$ jet production is biased towards being produced on the surface of the QGP, the second jet in these types of events has to then travel through the QGP to the far side. Studies of the far side reveal that the second jet is suppressed and takes on the properties of the medium it travels through. Studies show that the medium also responds to the passage of the second jet and heavy quarks are seen to lose less energy than light quarks.

\subsection{Colour transparency}

A study of colour transparency (CT) was presented by M. Holtrop (CLAS) [33]. The CT was studied in terms of nuclear transparency $T\left(A, Q^{2}\right)$ and is clearly observed in $\rho^{0}$ electroproduction, where $T\left(A, Q^{2}\right)$ was measured to increase with increasing $Q^{2}$, in a good agreement with predictions of the theoretical model by Kopelovich et al.

\subsection{Hadronisation in nuclear environment}

Recent results from HERMES were presented by Z. Akopov and Y. van Haarlem [34, 35]. Detailed studies of hadronisation on many nuclear targets were performed. Substantial nuclear attenuation was observed as a function of different kinematic variables. For the first time these studies were done using double-differential distributions. For the first time the formation length of the nuclear attenuation was studied. Absolute measurement of the $p_{T}$-broadening has been performed using different targets and hadron types for different kinematic variables.

\section{Jet production}

\subsection{Prompt photons}

S. Chekanov (ZEUS) presented recent results on prompt photons with an associated jet in photoproduction at HERA [36]. Prompt photon identification was based on conversion probabilities measured by a dedicated detector - Barrel Pre-sampler. Both PYTHIA and 
HERWIG fail to describe the shape and normalization of the cross sections. Best agreement is achieved using the $k_{T}$-factorization approach. An improved agreement with other NLO pQCD calculations was achieved by hardening the cut on the transverse momentum of the photon.

The H1 collaboration (talk by K. Müller) has performed prompt photon studies in DIS [37]. The Prompt photon signal was extracted using a log-likelihood approach based on a shower shape analysis. The measurements were done both for isolated photons and photons with jets. The LO MC models underestimate the data but the shapes are generally described. NLO pQCD calculations were not yet available.

O. Atramentov (DØ) presented inclusive photon+jet results in $p \bar{p}$ collisions [38]. Triple differential cross sections were measured as well as the ratios of these cross sections, significantly reducing the effect of experimental and theoretical correlated uncertainties. Four different photon+jet topologies were studied to explore different kinematic regions of the gluon distribution functions. For some kinematic regions quantitative deviations from current QCD predictions are observed.

\subsection{Jets photoproduction at HERA}

High- $E_{T}$ jets in photoproduction (PHP) measured by ZEUS and presented by H. Perrey show sensitivity to the gluon PDF and have the potential to further constrain the parton densities of the proton and photon [39]. None of the compared photon PDFs provided an adequate description of the ZEUS resolved data, although the direct enriched cross sections, which are less sensitive to the photon PDF, are in good agreement with NLO pQCD calculations.

The photoproduction of dijet events with a large rapidity gap between the jets was used by ZEUS to estimate the colour-singlet exchange contribution, as reported by A. Savin (ZEUS) [40]. Multi-jet photoproduction was used to study the properties of Multi-Parton Interactions (MPI) at HERA. The low-invariant-jet-mass region can only be described by adding the MPI to the MC simulation, thus providing a good testing ground for different MPI models. This information is very important for understanding the structure of underlying events at the LHC.

\subsection{Study of underlying events in DIS}

Further studies on underlying events at HERA were performed by H1 using the DIS data by measuring jets with low transverse momenta. S. Osman (H1) demonstrated in his talk, that the standard QCD MC models fail to describe the data in different azimuthal regions with respect to the leading jet [41]. Adding the MPI significantly improves the description at low $Q^{2}$ where resolved photon processes are expected to contribute, but still fails at high $Q^{2}$. Due to lack of NLO pQCD calculation suitable for this analysis no conclusion from high-order contribution was drawn.

\subsection{Charge current jets at HERA}

The first charge current (CC) ep jet analysis of the HERAII data was presented by H. Wolfe (ZEUS) [42]. For the first time the three- and four-jet events in CC DIS are analyzed. Inclusive jets and multi-jet cross sections were measured. Total polarized inclusive jet cross sections agree with predictions of the Standard Model. 


\subsection{Jets in $e e$}

An investigation of high momentum hadron and jet production by the OPAL collaboration was presented by A. Krasznahorkay [43]. Previously measured exclusive jet cross sections by L3 was not well reproduced by the pQCD NLO predictions. The new measurement by OPAL is well described by the theory. When compared in the same kinematic regime as L3, the OPAL and L3 measurement do not agree well and this difference has to be understood. The $p_{T}$ spectrum of hadrons is well described by the calculation except in the very high $p_{T}$ bin.

\subsection{Jets in $p \bar{p}$ and $p p$}

A preliminary measurement of the $b \bar{b}$ dijet production cross section and the angular correlation of the jets was presented by S. Vallecorsa (CDF) [44]. The NLO calculations describe the data well. The $b \bar{b}$ angular correlation shows that events are mainly produced by the flavour creation mechanism. The low $\Delta \phi$ tail suggests non-negligible contribution from other processes. It was demonstrated that the inclusion of the underlying event in the simulation significantly improves the description of the data.

M. D'Onofrio (CDF) reported on measurements of $W+$ jets and $Z+$ jets production cross sections at CDF [45]. Boson+jet production channels are fundamental to test pQCD, underlying events and new theoretical calculations. Comparisons to different calculations were shown. The size of non-perturbative corrections was also estimated. The measurement is important since it gives an estimate of background for new physics like top, Higgs and SUSY searches.

Jet production measurements was also reported by J. Cammin (DØ ) [46]. Good agreement with NLO pQCD predictions over a large $p_{T}$ range was demonstrated. Systematic uncertainties are dominated by the jet energy scale. Measurements of jets with a $\mu$ tag, used to identify Heavy Flavor jets, and $Z+$ jet production were also presented.

Prospects for inclusive jet cross section measurements with early data at ATLAS were presented by D. Clements [47]. Experimental errors are expected to be dominated by the jet energy scale. Theoretical errors at high $p_{T}$ are dominated by the uncertainty on the high-x gluon PDF.

\subsection{Inclusive jet production and jet correlations in DIS}

Recent results on angular correlations in three-jet events and jet substructure were presented by E. Ron (ZEUS) [48]. Fixed-order calculations, separated according to the colour configurations, were used to study the sensitivity of the angular distributions to the underlying gauge structure. Using different variables one can distinguish between contributions from triple-gluon vertexes in quark-induced and gluon-induced processes. The measurements are consistent with the admixture of colour configurations as predicted by $S U(3)$ and disfavour some other combinations. Subjet structure is reasonably described by the fixed-order QCD calculations and are consistent with the dominance of quark-induced processes.

Inclusive jet production in DIS at high $Q^{2}$ is a well established measurement and is well reproduced by different pQCD calculations. This is why this regime is used for precise QCD measurements and extraction of $\alpha_{S}$. An updated H1 analysis with improved precision was presented by T. Kluge (H1) [49]. The $\alpha_{S}$ fit was performed minimising the experimental uncertainty. A first extraction of $\alpha_{S}$ using the ratio of inclusive jet to the inclusive DIS 
cross sections was made which provides a more precise measurement. The ZEUS measurements were presented by T. Schoerner-Sadenius [50]. Together with inclusive cross sections ZEUS measured dependence of inclusive jet cross section on jet radius and used this measurement to extract the $\alpha_{s}$ value. The inclusive dijet sample was analyzed using combined HERAI and HERAII data, correspondent to integrated luminosity of $210 \mathrm{pb}^{-} 1$. For all these measurements the theoretical uncertainties still dominate.

Inclusive jet production cross section in the low $Q^{2}$ region, $5<Q^{2}<100 \mathrm{GeV}^{2}$, was presented by A.Baghdasaryan (H1) [51]. The analysis extends to lower $Q$ the similar analysis performed by $\mathrm{H} 1$ at high $Q^{2}$. Good agreement with NLO pQCD calculation was observed for $Q^{2}$ above $10 \mathrm{GeV}^{2}$. For further talks on jet production in the low- $x$ regime see forward jet production and HERA multi-jet production at low- $x$ and low- $Q^{2}$ presented during a special Structure Function WG session.

\section{Summary}

In addition to several formal theoretical developments, further experimental and theoretical contributions demonstrated great progress towards a more precise understanding of QCD final state production. Enormous progress in the theoretical calculations triggered by more precise data included a first resummation for QCD 3-jet production at Next-to-Leading Order which should provide a better description of dijet azimuthal correlation measurements both at HERA and the Tevatron. An additional dedicated session was held to discuss $\alpha_{S}$, where theoretical uncertainties dominate experimental measurements at HERA. NNLO calculations for 3 -jet production in $e^{+} e^{-}$were presented and are expected to lead to new fits for $\alpha_{s}$ with reduced theoretical uncertainties.

Future new physics searches at the LHC will rely on the most precise determinations of the parton density functions. HERA and Tevatron photon + jet production measurements, highlight regions where continuing improvements to theoretical models and inputs are still required and measurements of high $E_{T}$ forward dijet production at HERA and inclusive jet production at the Tevatron should also help further constrain these inputs. Progress towards a precision determination of unintegrated PDFs and a first determination of their intrinsic $k_{T}$ component was also presented at the meeting.

Tests of power corrections arising from hadronization effects have been extended to 3 -jet event shape distributions. Progress in perturbative evolution and hadronization issues, the role of the underlying event implementation in Monte Carlos, reducing model approximations for parton showers and issues that arise when Monte Carlos are tuned to non-global parameters were highlighted. The importance of multiple interaction modeling was demonstrated by HERA $\gamma p$ multijet production measurements and $b \bar{b}$ jet azimuthal correlations at the Tevatron. Improvements using a new flavor jet algorithm to theoretical uncertainties for heavy flavor jet production were demonstrated.

Recent exclusive final state measurements include an observation of two virtual photon annihilation final states at BaBar. Work in progress to resolve pentaquark issues currently show no further evidence for pentaquark states. A first measurement of the ratio of deuteron and antideuteron production rates in DIS at HERA is smaller than expected in the coalescence model. NLO pQCD predictions using improved fragmentation functions are found to describe the measured particle $p_{t}$ spectra at RHIC, however the effect of fragmentation functions is small and unable to account for observed differences to NLO pQCD predictions in scaled momentum spectra measurements in DIS at HERA. In collisions with heavy ions 
recent measurements at RHIC on the impact of Quark-Gluon plasma properties to particles passing through it were presented, together with first double differential nuclear attenuation measurements on a series of different nuclear targets at HERMES and color transparency effects which were reported in $\rho^{0}$ electroproduction measurements at CLAS. Further details for each individually presented topic can be found in the dedicated contributions to these proceedings.

\section{References}

[1] http://indico.cern. ch/contributionDisplay.py? contribId=18\&sessionId=2\&conf Id=9499

[2] A. Gehrmann, these proceedings; see also A. Gehrmann-De Ridder, T. Gehrmann, E. W. N. Glover and G. Heinrich, Nucl. Phys. Proc. Suppl. 160 (2006) 190 [arXiv:hep-ph/0607042].

[3] A. Gehrmann-De Ridder, T. Gehrmann and E. W. N. Glover, JHEP 0509 (2005) 056 [arXiv:hep$\mathrm{ph} / 0505111]$.

[4] R. Harlander, P. Kant, L.Mihaila, M. Steinhauser, these proceedings, arXiv:0706.2982 [hep-ph]; see also R. Harlander, P. Kant, L. Mihaila and M. Steinhauser, JHEP 0609 (2006) 053 [arXiv:hep-ph/0607240]; R. V. Harlander, D. R. T. Jones, P. Kant, L. Mihaila and M. Steinhauser, JHEP 0612 (2006) 024 [arXiv:hep-ph/0610206].

[5] J. Bluemleinand S. Klein, these proceedings, arXiv:0706.2426 [hep-ph]; see also J. Blumlein, Comput. Phys. Commun. 159 (2004) 19 [arXiv:hep-ph/0311046].

[6] N. Nikolaev, these proceedings; N. N. Nikolaev and W. Schafer, Phys. Rev. D 74 (2006) 074021 [arXiv:hep-ph/0607307].

[7] M. Dasgupta and G. P. Salam, Phys. Lett. B 512 (2001) 323 [arXiv:hep-ph/0104277].

[8] R. B. Appleby and M. H. Seymour, JHEP 0212 (2002) 063 [arXiv:hep-ph/0211426].

[9] Y. Delenda, R. Appleby, M. Dasgupta and A. Banfi, JHEP 0612 (2006) 044 [arXiv:hep-ph/0610242]. A. Banfi and M. Dasgupta, Phys. Lett. B 628 (2005) 49 [arXiv:hep-ph/0508159].

[10] M. Hansson [H1 Collaboration], Prepared for 14th International Workshop on Deep Inelastic Scattering (DIS 2006), Tsukuba, Japan, 20-24 Apr 2006.

[11] Y. Delenda, these proceedings, arXiv:0706.2172 [hep-ph].

[12] A. Banfi, these proceedings arXiv:0706.2722 [hep-ph]; see also A. Banfi, In the Proceedings of FRIF workshop on first principles non-perturbative QCD of hadron jets, LPTHE, Paris, France, 12-14 Jan 2006, pp B001 [arXiv:hep-ph/0605125].

[13] H. Jung and G. P. Salam, Eur. Phys. J. C 19 (2001) 351 [arXiv:hep-ph/0012143].

[14] H. Jung, these proceedings; see also H. Jung, A. V. Kotikov, A. V. Lipatov and N. P. Zotov, arXiv:hepph/0611093.

[15] Z. Nagy, Phys. Rev. Lett. 88, 122003 (2002) [arXiv:hep-ph/0110315]; Phys. Rev. D 68, 094002 (2003) [arXiv:hep-ph/0307268].

[16] G. Zanderighi, these proceedings, arXiv:0705.1937 [hep-ph]; see also A. Banfi, G. P. Salam and G. Zanderighi, arXiv:0704.2999 [hep-ph].

[17] M. Dasgupta, these proceedings, arXiv:0706.2630 [hep-ph]; see also A. Banfi, G. Corcella and M. Dasgupta, JHEP 0703 (2007) 050 [arXiv:hep-ph/0612282].

[18] L. Magnea, these proceedings.

[19] Z. Nagy, these proceedings; see also Z. Nagy and D. E. Soper, arXiv:0706.0017 [hep-ph].

[20] S. Visscher, these proceedings; see also J. Alwall et al., arXiv:0706.2334 [hep-ph].

[21] F. Maltoni, T. McElmurry, R. Putman and S. Willenbrock, arXiv:hep-ph/0703156.

[22] A. Vicini, these proceedings; see also C. M. Carloni Calame, G. Montagna, O. Nicrosini and A. Vicini, JHEP 0612 (2006) 016 [arXiv:hep-ph/0609170].

[23] K. Melnikov and F. Petriello, Phys. Rev. D 74 (2006) 114017 [arXiv:hep-ph/0609070]; C. Anastasiou, L. J. Dixon, K. Melnikov and F. Petriello, Phys. Rev. D 69 (2004) 094008 [arXiv:hep$\mathrm{ph} / 0312266]$.

[24] P. Rossi, these proceedings and references therein.

[25] M. Del Degan, these proceedings and references therein. 
[26] S. Chekanov, these proceedings and references therein.

[27] S. Saremi, these proceedings and references therein

[28] B. Levchenko, these proceedings and references therein.

[29] M. Heinz, these proceedings and references therein.

[30] B. Brzozowska, these proceedings and references therein.

[31] L. Pinera, these proceedings and references therein.

[32] C. Ogilvie, these proceedings and references therein.

[33] M. Holtrop, these proceedings and references therein.

[34] Z. Akopov, these proceedings and references therein.

[35] Y. Van Haarlem, these proceedings and references therein.

[36] S. Chekanov, these proceedings and references therein.

[37] K. Müller, these proceedings and references therein.

[38] O. Atramentov, these proceedings and references therein.

[39] H. Perrey, these proceedings and references therein.

[40] A. Savin, these proceedings and references therein.

[41] S. Osman, these proceedings and references therein.

[42] H. Wolfe, these proceedings and references therein.

[43] A. Krasznahorkay, these proceedings and references therein.

[44] S. Vallecorsa, these proceedings and references therein.

[45] M. D'Onofrio, these proceedings and references therein.

[46] J. Cammin, these proceedings and references therein.

[47] D. Clements, these proceedings and references therein.

[48] E. Ron, these proceedings and references therein.

[49] T. Kluge, these proceedings and references therein.

[50] T. Schoerner-Sadenius, these proceedings and references therein.

[51] A. Baghdasaryan, these proceedings and references therein. 